\title{
O guardião dos campos: um estudo sobre o comportamento do quero-quero Vanellus chilensis (Aves: Charadriiformes) no sul do Brasil
}

\author{
Henrique C. Delfino (1) \& Caio J. Carlos (1)
}

Laboratório de Sistemática e Ecologia de Aves e Mamíferos Marinhos, Departamento de Zoologia, Instituto de Biociências, Universidade Federal do Rio Grande do Sul (UFRGS). Av. Bento Gonçalves 9500, caixa postal 15007, 91501-970 Porto Alegre, RS, Brasil. (henrique.delfino@ufrgs.br)

\author{
Recebido 31 agosto 2019 \\ Aceito 22 de julho 2020 \\ Publicado 09 setembro 2020 \\ DOI 10.1590/1678-4766e2020022
}

\begin{abstract}
Guardian of the fields: a study on the behavior of the Southern Lapwing Vanellus chilensis (Aves: Charadriiformes) in Southern Brazil. The Southern Lapwing Vanellus chilensis (Molina, 1782) is a common bird in Rio Grande do Sul state, south Brazil, but is widely distributed across South America. It inhabits grasslands and other open habitats and it is known by a distinctive black, grey, and white coloration, a conspicuous vocalization and both aggressive and territorial behaviors. Although being recognized as the bird symbol of Rio Grande do Sul, no study about its behavior has yet been carried out in the state. Here, we aimed to describe and quantify the behavioral repertoire of Southern Lapwing. Between December of 2017 and November of 2018, we carried out observations in an eight-hectares grassland site in northern Rio Grande do Sul. We used ad libitum and animal focusing techniques to identify and record the frequency and duration of specific behaviors. An ethogram was elaborated, representing the 23 detected behaviors, grouped in five categories: locomotion, maintenance, feeding, socio-agonistic, and reproduction. Our analysis showed that most behaviors vary in both frequency and duration over the year. We detected high frequency and duration of social-agonistic and reproductive behaviors during the breeding season, while other categories, like feeding and maintenance, showed a decrease in the same period, with greater commitment to protect the eggs and chicks. We also recorded three types of vocalization with different characteristics, one of which occurs exclusively during the breeding season. The results of our study allowed the identification and quantification of $V$. chilensis in southern Brazil, thus helping in understanding the relations between the species and its habitats in the region.
\end{abstract}

KEYWORDS. Ethology; interactions; reproduction; vocalization; agonistic behavior.

RESUMO. O quero-quero Vanellus chilensis (Molina, 1782) é uma ave típica do Estado do Rio Grande do Sul, mas possui ampla distribuição geográfica nos campos da América do Sul, sendo conhecido pelo canto imponente e pelos comportamentos relacionados com a agressividade e a territorialidade. Apesar de ser reconhecido como animal símbolo do estado, nenhum trabalho acerca dos comportamentos dessa ave foi desenvolvido no estado. Este trabalho teve como objetivo qualificar e quantificar o repertório comportamental de $V$. chilensis. Observações dos comportamentos dessa ave foram feitas em uma área de campo com oito hectares, no município de Torres, RS, entre dezembro de 2017 e novembro de 2018, totalizando 143 horas de observação. Utilizou-se a metodologia de observação de todas as ocorrências e observação de animal focal, coletando-se dados de frequência de ocorrência e duração de cada comportamento. Com os dados construiu-se um etograma. Registrou-se 23 comportamentos divididos em cinco categorias: locomoção, manutenção, alimentação, social-agonista e reprodução. Identificaram-se três formas de vocalização distintas em sonoridade, duração e ocasião, o que sugere diferentes funções para cada uma. As análises comprovaram a variação da maior parte dos comportamentos ao longo dos meses do ano, tanto em frequência como em duração. Constatou-se o aumento dos comportamentos sociais-agonistas e reprodutivos durante a estação reprodutiva, enquanto outras categorias como alimentação e manutenção apresentaram diminuição nesse período, havendo maior empenho dos animais na proteção dos ninhos e filhotes. O trabalho permitiu a caracterização dos comportamentos de $V$. chilensis no sul do Brasil, auxiliando no entendimento das relações entre espécie e ambiente típico da região.

PALAVRAS-CHAVE. Etologia; interações; reprodução; vocalização; comportamento agonístico.

O quero-quero Vanellus chilensis (Molina, 1782) é uma ave Charadriiformes de porte médio com $35-37 \mathrm{~cm}$ de comprimento total e $255-277 \mathrm{~g}$, que ocorre principalmente em regiões campestres, mas também em áreas costeiras e ambientes alagadiços, desde a América Central até o sul da América do Sul (Del Hoyo et al., 1996; Ridgely \& GREENFIELD, 2001). Recentemente, a espécie vem expandindo sua distribuição em direção à bacia amazônica, provavelmente por causa do desmatamento nessa região (SANTOS, 2010).

A espécie é frequentemente encontrada em bandos de quatro a 80 indivíduos (CosTA, 2002), mas agregações com mais de uma centena de indivíduos já foram observadas (MARUYAMA et al., 2010). No período de reprodução, que no Brasil geralmente ocorre de agosto a dezembro (BELTON, 1994; Sick, 1997), os indivíduos tendem a se separar e formar duplas ou trios reprodutivos, podendo ocorrer tanto a monogamia quanto a poligamia (SARACURA et al., 2008). Apesar do quero-quero empregar pouco esforço na construção do ninho (Simon \& PaCheCo, 2005), é conhecido por ser territorial e apresentar uma variedade de comportamentos de cuidado parental, que inclui defesa do ninho e filhotes (COSTA, 2002), além da termorregulação (SARACURA et al., 2008). 
Os comportamentos agressivos intra- e interespecíficos do quero-quero podem ser explicados pela intensa exposição das aves em ambientes de campo às interações com outros animais, especialmente as aves de rapina, mas também de outras pequenas aves da região e animais de criação doméstica (Costa, 1994b), além de outros indivíduos da mesma espécie e do ser humano. Comportamentos agonísticos do queroquero variam em tipo, frequência e intensidade, de acordo com a época do ano e fase do período reprodutivo (CosTA, 2002), havendo também influência do tipo do invasor e da distância entre este e o quero-quero (ElLIOT, 1985). Esses comportamentos também parecem influenciar diretamente a sobrevivência de filhotes e a manutenção dos tamanhos populacionais de quero-queros (SARACURA et al., 2008; SANTOS \& MACEDO, 2011).

A etologia é a área da ciência que se dedica ao estudo do comportamento animal e investiga questões em torno das relações entre comportamento, ambiente e indivíduo (LoRENZ, 1995). Dessa forma, o estudo dessas interações e desses comportamentos se mostra essencial para se entender a dinâmica grupal e populacional do quero-quero, ajudando a identificar e categorizar fatores chaves relacionados com a manutenção e evolução dessas características tão peculiares. Pesquisas nesse sentido já foram realizadas com o abibecomum $V$. vanellus (Linnaeus, 1758) (BARNARD \& STEPHENS, 1981; Buitron, 1983; THOMPSON, 1983, 1986; ThOMPSON \& BARNARD, 1983, 1984; THOMPSON \& LENDREN, 1985; ElLIOT, 1985; IVERSEN, 1986; BeINTEMA \& VisSER, 1989a,b; Galbraith, 1989; HesP \& BARNARD, 1989; KIRBy \& GREEN, 1991; McMahon, 1996), abibe-coroado V. coronatus (Boddaert, 1783), abibe-d'asa-negra Vanellus melanopterus (Cretzschmar, 1829), abibe-d'asa-negra-pequeno V. lugubris (Lesson, 1826) (WARD, 1989), abibe-preto-e-branco $V$. armatus (Burchell, 1822) (ThomAs, 1983), abibe-de-facesbrancas V. crassirostris (Hartlaub, 1855) (WALTERS, 1979) e abibe-vermelho $V$. indicus (Boddaert, 1783) (KALSI \& KHERA, 1987), mas todos no hemisfério norte.

Em relação ao quero-quero, alguns trabalhos semelhantes já foram desenvolvidos no Brasil (CosTA, 1994a,b, 2002); entretanto, nenhum deles em território sul-rio-grandense e nenhum com abordagem qualitativa e quantitativa. Há, dessa forma, poucas informações acerca do comportamento dessa espécie nos Campos Sulinos, bioma que abrange a maior parte do estado (PIllar et al., 2009). Assim, faz-se necessário conhecer melhor a etologia da ave símbolo do Rio Grande do Sul, presente na cultura popular e tradicionalista do estado, mas pouco investigada do ponto de vista científico. Além disso, informações sobre o comportamento do quero-quero podem auxiliar no manejo desses animais em áreas urbanas, como campos de futebol, praças e parques, problemática presente e frequente nas cidades do sul e do Brasil.

Os objetivos deste trabalho, portanto, são: 1) qualificar os comportamentos de quero-quero no Rio Grande do Sul, possibilitando a formação de um banco de referência (catálogo); 2) quantificar os comportamentos e as variações desses ao longo de um ano, evidenciando as possíveis mudanças comportamentos entre a estação reprodutiva e não reprodutiva.

\section{MATERIAL E MÉTODOS}

Área de estudo. As observações foram conduzidas no município de Torres, Rio Grande do Sul, nas proximidades do Parque Estadual da Itapeva, em um campo aberto de aproximadamente 8 ha. A área apresenta vegetação campestre nativa e baixa, com o domínio de gramíneas e a ausência de árvores de grande porte, havendo também algumas formações arbustivas. A pastagem é sazonal, tipicamente de verão, e é facilmente alagável, possuindo áreas úmidas e banhados que se mantêm durante praticamente o ano todo (HCD, observ. pess.).

A região é frequentemente utilizada como local para abrigo e criação de animais domésticos e para pecuária extensiva, além de possuir alto grau de antropização, por causa do antigo uso da área para descarte de material de construção e lixo, sofrendo também com os interesses imobiliários nas proximidades. $\mathrm{Na}$ área, convivem com os quero-queros, equinos, bovinos, cães, galinhas, pequenas aves e outros animais, além do constante fluxo de seres humanos a pé ou motorizados, devido à passagem de uma estrada que corta a zona de estudo (HCD, observ. pess.).

A área foi escolhida por dois motivos principais: a presença abundante e constante de quero-queros durante todo o ano, que na estação não-reprodutiva utilizam a área para forrageio e alimentação, e na estação reprodutiva, escolhem o terreno como zona de nidificação, onde crescem os filhotes; e pelo fácil acesso à área a partir do município de Torres.

Amostragem. Em dezembro de 2017 foram feitos estudos preliminares, segundo o método de observação de todas as ocorrências (ALtMANN, 1974) para qualificar e formar um banco de dados dos comportamentos dos indivíduos que habitavam a área de estudo. Essas observações duraram cerca de duas horas diárias, perfazendo um total de dez horas. Na qualificação dos comportamentos de interação, foram utilizadas como referência as categorias descritas na literatura (e.g., Costa, 2002).

As observações subsequentes foram conduzidas entre dezembro de 2017 e novembro de 2018, totalizando 143 horas. Utilizou-se o método de animal focal (AltmanN, 1974), com sessões de observação de 10 minutos e intervalos de 5 minutos. As observações foram realizadas pela manhã, entre $8 \mathrm{~h} 00$ e $10 \mathrm{~h} 00$, e à tarde, entre $16 \mathrm{~h} 00$ e $18 \mathrm{~h} 00$, sempre respeitando-se um período de habituação de 30 minutos. Os indivíduos foram observados a uma distância de 60-80 m, com binóculos e câmeras fotográficas digitais, sem haver a manipulação ou o anilhamento das aves.

Análise dos dados. Com base nos dados comportamentais coletados e organizados de acordo com cada tipo de comportamento, foram construídos etogramas a partir dos quais foi possível quantificar a duração média de atividade, períodos de atividades e interações mais intensas, além de destacar a mudança de comportamento ao longo do tempo e do espaço (Del-Claro, 2004). 
Para testar se houve variação na frequência dos comportamentos ao longo dos meses do ano, foram utilizados teste $\mathrm{G}$ para o conjunto geral dos dados e testes de qui-quadrado $\left(\mathrm{X}^{2}\right)$ para cada categoria comportamental observada, bem como para as frequências relativas de cada um dos comportamentos registrados. Para testar se houve diferença na duração dos comportamentos ao longo do ano, foi utilizado o teste não paramétrico de Kruskal-Wallis, já que os dados não seguiam uma distribuição normal (ELLISON \& Gotelli, 2011). As análises foram realizadas no programa R (R Core TeAm, 2015) e BIOESTAT ${ }^{\circledR}$ (Ayres et al., 2007).

\section{RESULTADOS}

Durante o estudo foram identificados e descritos 23 comportamentos, divididos em 5 categorias: alimentação, manutenção, locomoção, reprodução e social-agonista.

\section{Alimentação}

1. Comer: o animal observa o local onde está parado, em posição neutra, até identificar o alimento, fazendo assim um movimento de abaixar o bico em direção ao solo para apreendê-lo. Por vezes, o animal pode movimentar um dos pés sobre o solo para aumentar a exposição do alimento e facilitar a captura (Fig. 1).

2. Tremular dos pés (Foot-Trembring): o animal se apoia em um dos pés enquanto movimenta a outra sobre a pastagem, em movimento trêmulo repetitivo, batendo-as sobre o solo seco, à procura de presas, que assim são mais facilmente capturadas. Esse comportamento geralmente é seguido do comportamento de alimentação e está presente em outras espécies da mesma família, como na batuíra-de-bando Charadrius semipalmatus (Bonaparte, 1825) (CESTARI, 2009).

3. Beber: o animal se aproxima de uma fonte de água, geralmente uma poça, e inclina o pescoço e o bico em direção ao líquido, posteriormente elevando a cabeça para facilitar a deglutição (Fig. 2).

4. Defecar: sobre os dois pés, em posição neutra ou em movimento lento, o animal movimenta as penas da cauda de forma breve, elevando-as e eliminando o excremento. Rapidamente volta para a posição anterior.

\section{Locomoção}

1. Andar: o animal estabelece uma passada lenta e ritmada, com o pescoço elevado e com a cabeça projetada para frente. Geralmente, esse tipo de deslocamento ocorre entre pequenas distâncias e o animal com frequência para durante a caminhada, em posição neutra.

2. Correr: o animal posiciona a cabeça à frente do corpo e dá passadas rápidas em direção a um local determinado, geralmente em curtas distâncias (Fig. 3).

3. Voar: o animal inclina-se, abre e, posteriormente, bate as asas para pegar impulso e conseguir entrar em voo. No ar, o animal movimenta as asas repetidas vezes para alterar velocidade e altura de voo. Esse tipo de locomoção pode ocorrer entre pequenas e longas distâncias, geralmente entre um local de alimentação e outro.

4. Caminhar furtivo: o animal movimenta-se muito lentamente, com o pescoço abaixado e o bico próximo ao solo, geralmente movimentando-se após eventos de alimentação, ou em direção a outro indivíduo do mesmo grupo/bando; raramente utilizado durante interações (Fig. 4).

\section{Manutenção}

1. Posição neutra: o animal permanece ereto, com as asas junto ao corpo, pescoço em posição natural e o bico fechado, geralmente movimentando a cabeça ligeiramente para observar os arredores. Por vezes, pode ocorrer o recolhimento de uma das pernas, com alternância (Fig. 5).

2. Higienização das penas: o animal realiza o movimento de limpeza das penas com auxílio do bico, geralmente iniciando o processo nas penas da cauda e asas para, posteriormente, realizar a limpeza do dorso e região peitoral. As penas longas geralmente são alisadas, da base para as extremidades, enquanto debica as penas menores. Nos períodos de chuva, esse comportamento também é importante e contribui para a impermeabilização das penas do animal (Fig. 6).

3. Posição de descanso: o animal se abaixa no chão, escondendo as duas pernas sobre o corpo e encurtando o pescoço levemente, podendo também o apoiar sobre a asa (Fig. 7).

4. Posição de termorregulação: o animal, a partir da posição neutra, eriça as penas por um tempo determinado e encurta o pescoço, permanecendo imóvel. Esse comportamento é muito comum durante dias com vento forte, ou no período matutino em dias frios. (Fig. 8).

5. Espreguiçar-se: o animal ergue o corpo e distende o pescoço, esticando também as pernas e as asas, movimentando toda a musculatura corporal. Esse comportamento ocorre geralmente após as posições neutras ou de descanso, quando o animal permanece muito tempo numa mesma posição. Por vezes, o animal também estica as asas após pousar de um voo, antes de guardá-las junto ao corpo.

\section{Social-agonista}

1. Posição de alerta: a partir da posição neutra, o animal estende o pescoço e gira a cabeça para os lados, com o objetivo de observar seu território e possíveis invasores. Frequentemente executado juntamente com a vocalização de tipo 1, ou territorial (Fig. 9).

2. Posição de fuga ou distração: o animal, ao encontrar/ detectar um invasor, corre rapidamente para longe com a cabeça baixa e o pescoço levemente encolhido. Raramente acompanhada de vocalização (Fig. 10).

3. Posição de ameaça I ou de preparação: o animal, quando identifica um invasor próximo, abaixa o pescoço em direção ao solo, indo em direção ao outro indivíduo, com frequência vocalizando do tipo II ou de ameaça, abrindo levemente as asas ao se aproximar. Também é a posição que antecede a decolagem para um possível ataque aéreo (Fig. 14). 


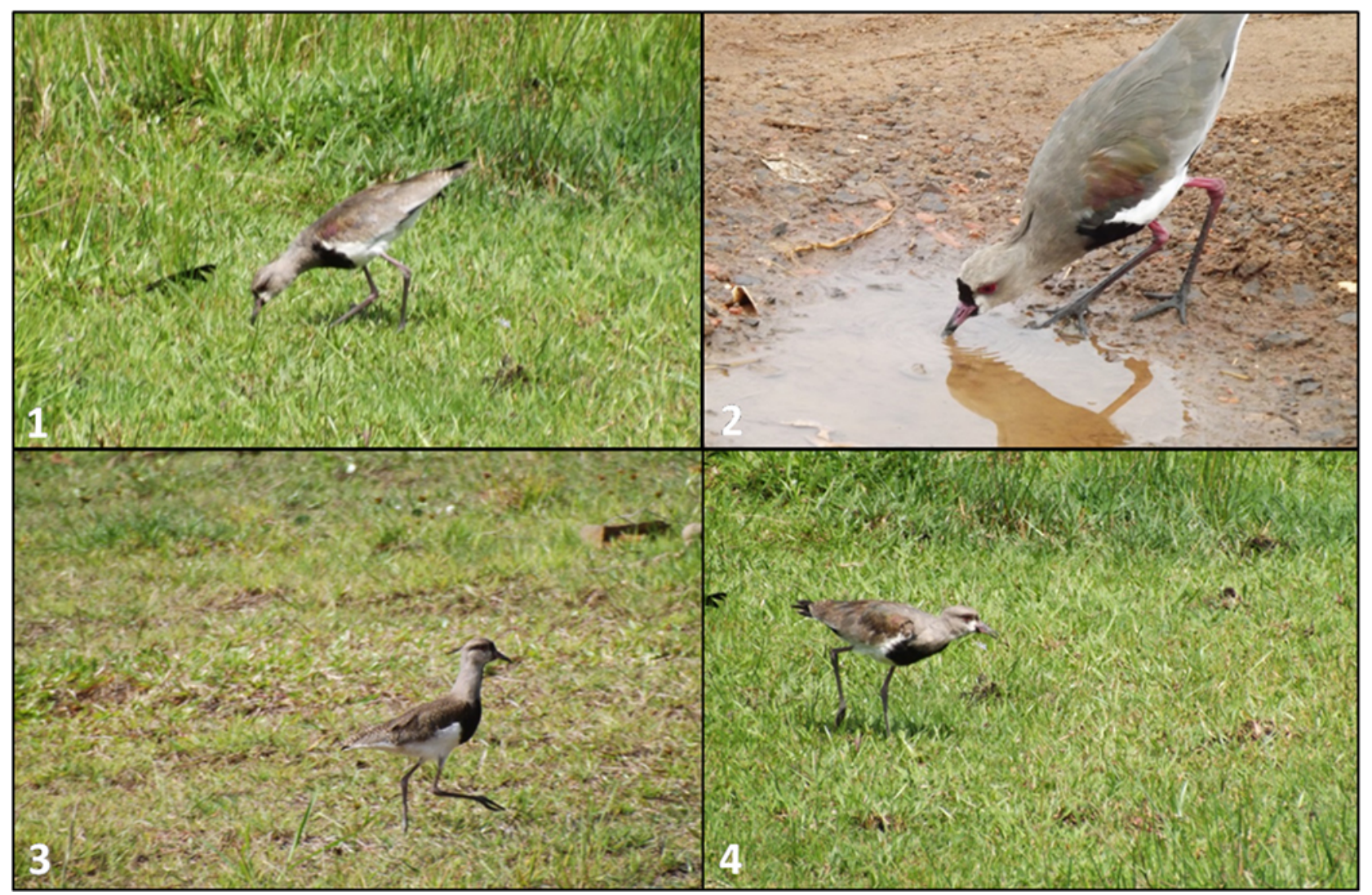

Figs 1-4. Alguns comportamentos observados em Vanellus chilensis para as categorias Locomoção e Alimentação: 1, comer; 2, beber; 3, correr; 4, andar furtivo. Imagens: HCD.

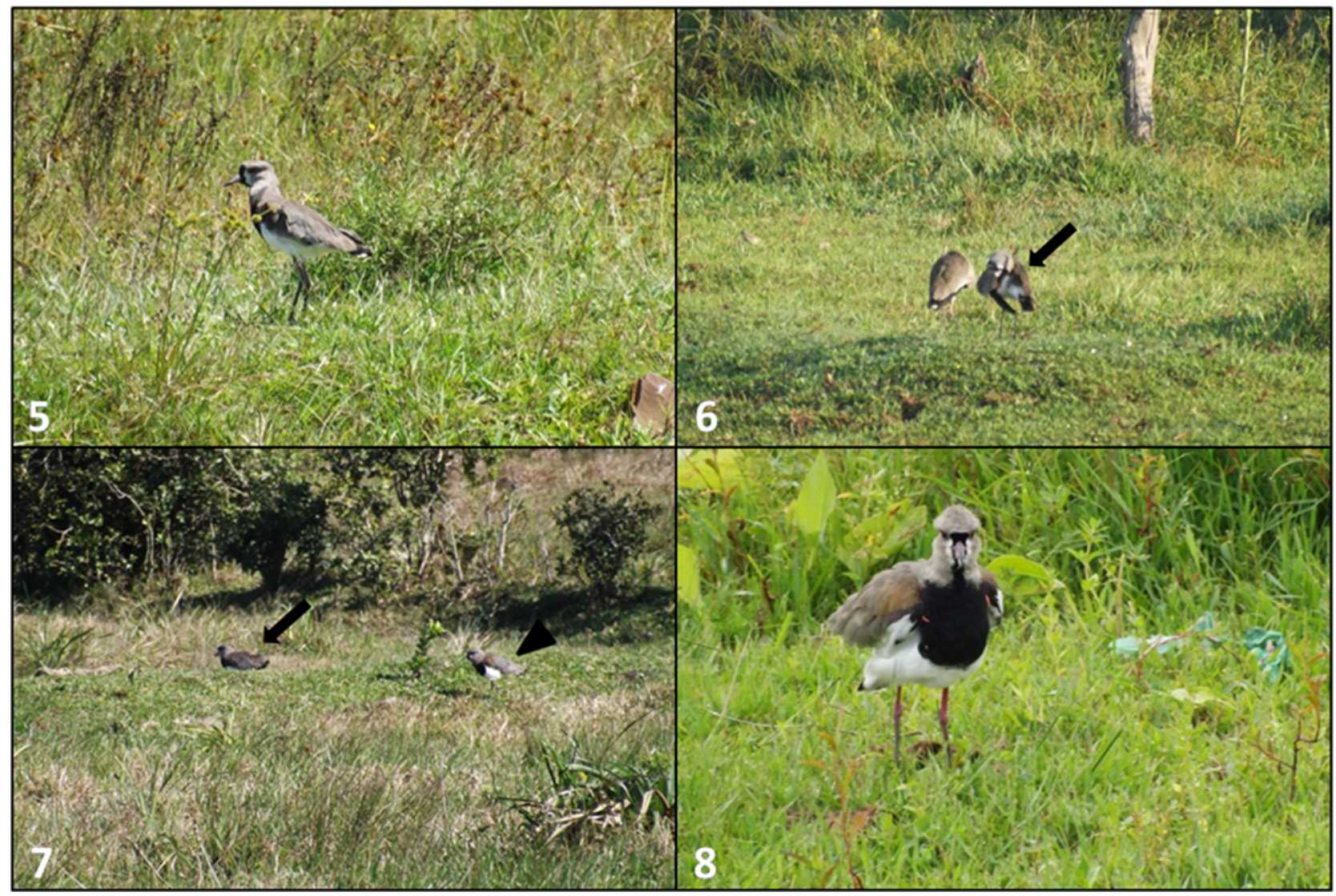

Figs 5-8. Alguns comportamentos observados em Vanellus chilensis relacionados à categoria de Manutenção: 5, posição neutra; 6, higienização das penas (apontado pela seta); 7, posição de descanso (apontada pela seta) e posição neutra (apontada pela cabeça de seta); 8, posição de termorregulação. Foto: HCD. 
4. Posição de ameaça II ou de ameaça frontal: o animal se desloca em direção ao invasor com a cabeça baixa e, ao se aproximar, eleva a cabeça e o pescoço rapidamente, estufando o peito e vocalizando fortemente. Esse comportamento geralmente ocorre em interações intraespecíficas ou com outras aves, sempre no meio terrestre (Fig. 11).

5. Ataque aéreo: a partir da posição de ameaça I, o animal decola e sobrevoa o invasor com voos rasantes e circulares e com as asas totalmente estendidas, deixando os esporões expostos, com forte vocalização do tipo II, aproximando-se do outro animal, mas raramente entrando em contato físico. Geralmente, esse comportamento é realizado em grupos de 2-5 indivíduos (Fig. 13).
6. Posição de agachamento: o animal, geralmente juvenil, quando em situações de risco ou estresse, agachase sobre o solo, escondendo ambas as pernas sobre o corpo, encolhendo o pescoço, numa clara tentativa de se tornar menos visível para o possível predador ou invasor. Geralmente não associado com vocalização (Fig. 12).

7. Posição de proteção dos ovos: na estação reprodutiva, quando se depara com um invasor muito próximo do ninho com os ovos, um dos indivíduos do bando posicionase sobre os ovos e abre as asas brevemente, expondo os esporões, com forte vocalização. Os demais indivíduos do grupo reprodutivo geralmente estão envolvidos no ataque aéreo.

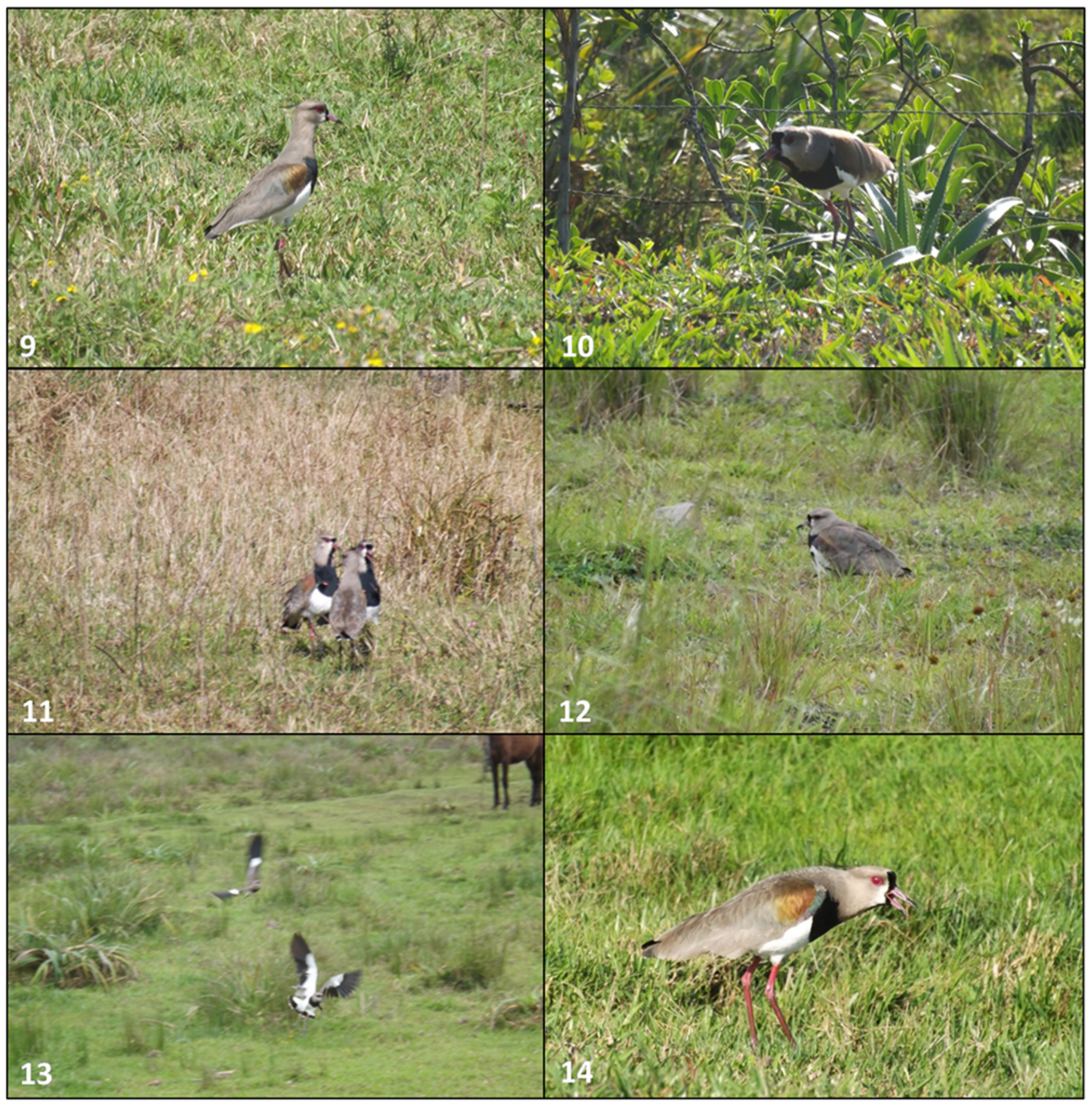

Figs. 9-14. Alguns comportamentos observados em Vanellus chilensis na categoria Social-Agonísta: 9, posição de alerta; 10, Posição de fuga ou distração; 11, Posição de ameaça II ou ameaça frontal; 12, Posição de agachamento; 13, Ataque aéreo; 14, Posição de ameaça I ou preparação. Foto: HCD. 
8. Posição de proteção dos filhotes: na estação reprodutiva, com os filhotes presentes, a ave corre em direção a eles e vocaliza com intensidade com o pescoço baixo e asas levemente abertas, protegendo-os sob as asas ou próximo ao seu corpo.

\section{Reprodutivos}

1. Cortejo: um grupo de 3-4 indivíduos se aproximam de outro indivíduo da mesma espécie com o peito estufado e com vocalização do tipo 3 ou reprodutiva, expondo os esporões. Observados nos meses do início da entrada da estação reprodutiva. Não há na literatura padrões de cortejo descritos para o quero-quero, mas os comportamentos observados condizem com os observados para outras espécies do gênero (MACLEAN, 1972).

2. Cópula: a fêmea e o macho interagem brevemente através de vocalização do tipo 3 e a fêmea inclina o pescoço em direção ao solo e eleva a cauda, com o macho posicionando-se sobre ela para efetuar a cópula.

3. Aninhamento: na presença de filhotes, e possivelmente, também dos ovos, quando em condições climáticas adversas, como vento forte e chuvas torrenciais, um dos indivíduos abriga os filhotes sob as asas, protegendoos. Durante situações de ataque ou com a aproximação de algum invasor próximo aos ninhos, este comportamento também era realizado por um dos indivíduos adultos.

Quanto às vocalizações, foram observados, registrados e analisados três diferentes tipos de vocalização, que diferiam em sonoridade, duração, frequência de ocorrência e ocasião em que eram emitidas:

1. Vocalização 1 ou de alerta: o quero-quero, geralmente em posição de alerta, emite um canto enfático e estereotipado de keeh keeh keeh (XC492891; Delfino, 2019a) quando em contato com outros indivíduos da mesma espécie e pequenas aves, bem como outros invasores maiores, ainda que com menor frequência. Está associada a interações intraespecíficas e territoriais;

2. Vocalização 2 ou de ameaça: o animal, geralmente, em posição de ameaça I, ameaça II ou de ataque aéreo emite kweh kweh kweh (XC492892; Delfino, 2019b) canto característico da ave, na tentativa de afastar invasores, como cães e humanos. Está mais relacionada com interações agonísticas e de predação;

3. Vocalização 3 ou reprodutiva: tipo de vocalização exclusivamente intraespecífico, com a ave repetindo várias vezes keh keh keh keh (XC492895; Delfino, 2019c), geralmente em grupo e na posição de cortejo. É exclusivo da estação reprodutiva do animal.

Foram registrados 21.018 eventos comportamentais ao longo do estudo. Os comportamentos mais frequentes foram os de Andar ( $\mathrm{n}=5.037)$, Comer $(\mathrm{n}=4.583)$ e Posição Neutra ( $\mathrm{n}=2.983)$, todos esses ocorrendo ao longo do ano. Alguns comportamentos, entretanto, foram observados somente na estação reprodutiva, como a corte $(\mathrm{n}=5)$, a cópula $(n=6)$ e o aninhamento $(n=19)$. $O$ teste $G$ indicou diferença na frequência das categorias ao longo dos meses $(p<0,001)$. Aplicando-se o qui-quadrado para cada categoria, constatouse que apenas as categorias sociais-agonistas, de vocalização e de reprodução apresentaram variação significativa nas frequências ao longo do ano $(\mathrm{p}<0,05)$, havendo aumento principalmente quanto aos comportamentos sociais-agonistas, entre os meses de julho a novembro (Fig. 15).

Quanto à duração, os comportamentos mais longos foram os de ataque aéreo (média $=224,8 \mathrm{~s}$ ) e a posição de descanso (média=104,9s). O teste de Kruskal-Wallis indicou que todas as categorias comportamentais apresentaram uma variação significativa na duração ao longo dos meses, em especial as categorias social-agonista $(p<0,001)$ e de vocalização $(p<0,001)$, que expressaram aumento considerável no início da estação reprodutiva (Fig. 16).

A partir da análise estatística foi possível constatar que a duração média das três vocalizações diferiu entre as estações não-reprodutiva e reprodutiva $(\mathrm{p}<0,001)$, indicando aumento da duração média dos cantos durante os meses de junho a novembro $(\mathrm{p}<0,001)$. Além disso, houve uma mudança na frequência de ocorrência de cada tipo de vocalização durante este período $(\mathrm{p}=0,006)$, que passaram a ser mais frequentes.

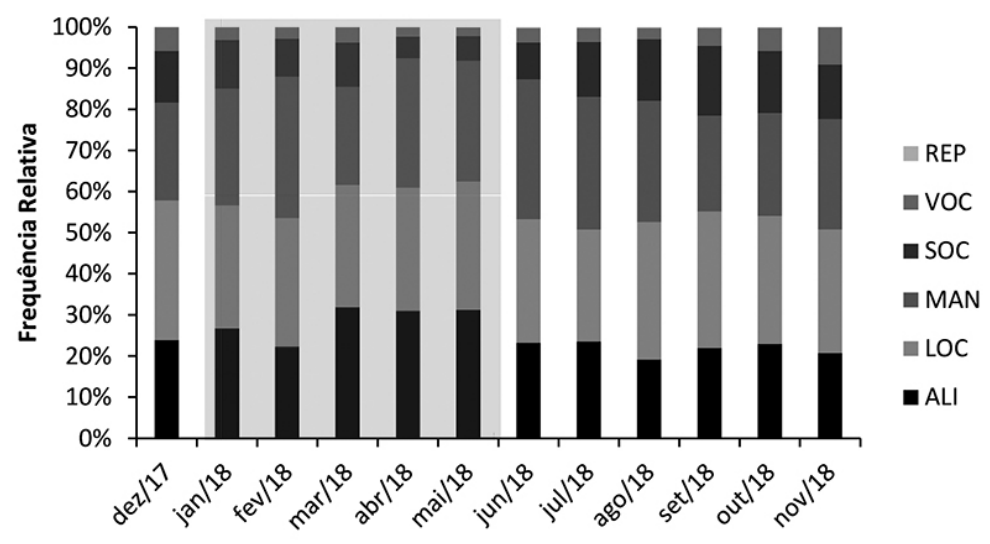

Fig. 15. Frequência relativa das categorias comportamentais de Vanellus chilensis ao longo do ano. Área em destaque representa a estação não-reprodutiva do animal (REP, Reprodução; VOC, Vocalização; SOC, Social-agonísta; MAN, Manutenção; LOC, Locomoção; ALI, Alimentação). 


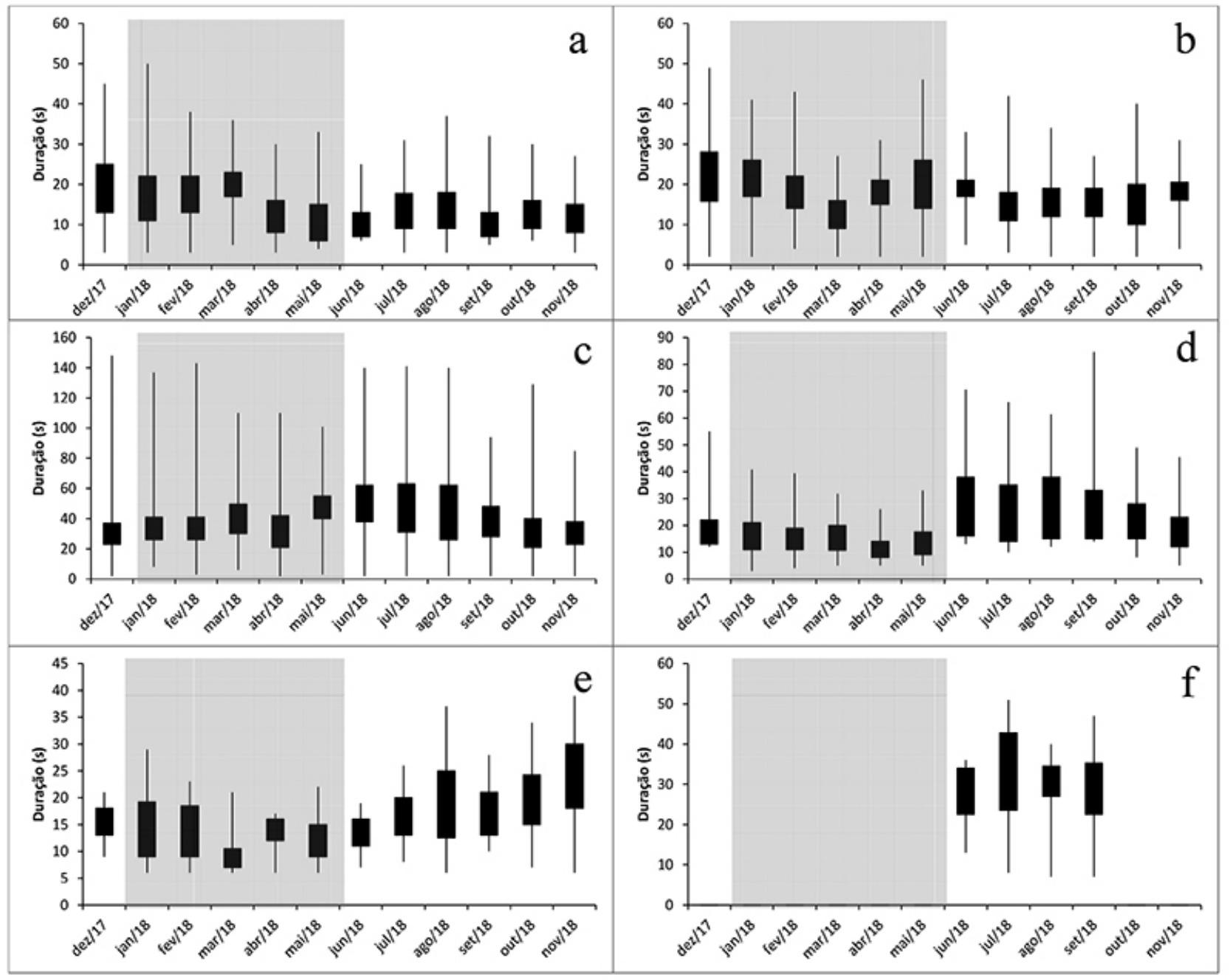

Fig. 16. Variação na duração de cada categoria comportamental de Vanellus chilensis ao longo do ano. a) Alimentação; b) Locomoção; c) Manutenção; d) Social-Agonísta; e) Vocalizações; f) Reprodução. Área em destaque representa a estação não-reprodutiva da ave, as barras representam o intervalo entre o primeiro quartil e o terceiro quartil, enquanto as linhas representam os valores máximo e mínimo.

\section{DISCUSSĀO}

O quero-quero é uma espécie conhecida por seu comportamento agressivo, este relacionado com a territorialidade e interações agonistas (CostA, 2002). Mas, assim como outras aves, também possui uma grande variedade comportamental quando se refere à manutenção, alimentação e locomoção (BAKHTURINA, 2016). Durante a estação reprodutiva no Rio Grande do Sul, principalmente entre agosto e setembro, houve um aumento no repertório comportamental pela inclusão dos comportamentos relacionados à reprodução, ainda que estes sejam menos frequentes.

Levando-se em consideração os resultados, verificouse que os indivíduos passam a maior parte do tempo realizando comportamentos relacionados com manutenção, locomoção e alimentação, assim como ocorre com outras Charadriidae, tais como a batuíra-de-bando C. semipalmatus, o maçaricopintado Actitis macularius (Linnaeus, 1766), o maçaricode-sobre-branco Calidris fuscicollis (Vieillot, 1819) e o maçarico-branco Calidris alba (Pallas, 1764) (WALTERS, 1982). Mas especificamente, os comportamentos de andar e alimentação apresentaram frequência elevada entre janeiro e junho, período não reprodutivo da ave, o que condiz com a tendência ao uso da área para forrageio nos meses de verão e outono (BeLton, 1994). Nesse período do ano, há desenvolvimento mais intenso da pastagem e maior riqueza e diversidade de artrópodes no solo (COLEMAN \& Crossley, 1995), principal recurso alimentar do quero-quero (DeL Hoyo et al., 2013).

Comportamentos relacionados à manutenção, especialmente os de termorregulação e à posição de descanso aumentaram de frequência e duração entre os meses de maio e agosto, possivelmente por causa da redução das temperaturas no fim do outono e no inverno, que gera uma menor atividade do animal quanto à alimentação e locomoção (MARSH \& DAwson, 1989). Nesse período do ano, a ave passa a destinar mais energia para as interações territoriais e para os aspectos reprodutivos (Plummer et al., 2013). A sazonalidade da pastagem e a presença, abundância e diversidade de recursos 
alimentares, bem como a variação desses fatores ao longo do ano, podem estar relacionados diretamente com aspectos comportamentais do animal (WILSON, 1975), bem como com características climáticas específicas da Região Sul, apesar de mais estudos serem necessários nesse aspecto.

Ficou evidente o extenso período de tempo destinado para comportamentos sociais, especialmente os que envolvem as interações agonistas. Resultados semelhantes foram encontrados para outras espécies de Vanellus (WALTERS, 1979; Thomas, 1983; WARD, 1989) e de Charadriidae (WALTERs, 1982). Além disso, o quero-quero apresenta mais comportamentos sociais e agonistas do que outras aves não proximamente relacionadas; por exemplo, o carcará Caracara plancus (Miller, 1777) (Falconidae) (OliveIRA et al., 2014), a garça-branca-grande Casmerodius albus (Linnaeus, 1758) (Ardeidae) (Henrique \& Piratelli, 2008) e o chupim Molothrus bonariensis (Gmelin, 1789) (Icteridae) (Porto \& Piratelli, 2005), todas presentes na região.

Os comportamentos observados condizem com aqueles já registrados na literatura (COSTA, 2002), sendo o mais frequente a posição de alerta, com poucas observações de ataques diretos. Entretanto, exibições de ataques aéreos aumentaram durante a estação reprodutiva, bem como a duração desses eventos comportamentais, indicando um maior gasto energético na realização destes comportamentos durante essa época, possivelmente para defender territórios e filhotes (COSTA, 2002).

Alguns comportamentos sócio-agonísticos também mudaram seu padrão de ocorrência ao longo do ano. As exibições de fuga e de agachamento passaram a ser mais comum nas estações não reprodutivas e sua duração variou ao longo do ano, evidenciando uma distinta fase de distanciamento não confrontativo, onde os indivíduos evitam interações e concentram-se quase que exclusivamente no forrageio e alimentação (CosTA, 2002). Já a exibição de ameaça teve um aumento considerável na frequência e duração durante o período de nidificação, bem como os comportamentos de proteção dos ovos e dos filhotes, que são exclusivos dessa época do ano. Isso marca outra fase comportamental da ave: a confrontativa. Nessa fase, também observada em outros Charadriiformes (MACLEAN, 1972), a ave emprega sua energia na proteção da ninhada e do território, buscando garantir maior sobrevivência da prole e consequentemente aumentando seu próprio sucesso reprodutivo (MOLLER, 2000).

Registrou-se três tipos de vocalização distintas em sonoridade, duração e ocasião, o que sugere diferentes funções para cada uma: uma territorial, uma de ameaça e outra relacionada à reprodução, observado somente entre agosto e novembro. Esses resultados são similares aos encontrados por Kumar \& SHARMa (2011), que registraram três tipos de vocalização para outras espécies de Vanellus: a vocalização territorial, a de ameaça e o canto "tit-tit", associado com a corte e à reprodução. A duração e a frequência das vocalizações variaram ao longo dos meses do ano, principalmente durante a estação reprodutiva. Essa mudança comportamental se deve à disputa de fêmeas $\mathrm{e}$ a formação dos territórios de nidificação nessa época, o que explica maior frequência e duração de vocalizações relacionadas às interações intraespecíficas (CosTA, 2002), com uma maior necessidade de defender, inicialmente, os ninhos e posteriormente os ovos e filhotes de invasores e predadores, indicando a importância das emissões sonoras nas relações sociais desses animais, como a formação dos casais e a defesa dos territórios, dos ovos e dos filhotes (SARACURA et al., 2008). No período não reprodutivo, o indivíduo está focado na autodefesa e a vocalização passa a ser curta e pouco frequente, já que a exibição (display) de fuga é a mais utilizada.

Apesar da maior parte das aves onívoras forrageiras demonstrarem comportamentos não agressivos com maior frequência (WILSON, 1975), esse não parece ser o caso do quero-quero. Este trabalho demonstrou o complexo repertório comportamental e de interações que essa espécie possui e sua relação com a época de seu ciclo de vida, período do ano e fatores ambientais. A partir dos resultados é possível formar um panorama do comportamento dessa ave típica do sul do Brasil, ainda pouco estudada no país, bem como identificar fatores que influenciam diretamente na forma como esses comportamentos se dão na natureza.

Fatores ambientais, como clima e vegetação do sul do Brasil, podem influenciar diretamente o comportamento da ave em campo nativo e tornar a etologia da espécie única nessa região, indicando que aspectos comportamentais do quero-quero sejam mais complexos do que parecem, necessitando de mais estudos nesse âmbito. É preciso entender como os mecanismos comportamentais dessa ave evoluíram ao longo da história de vida da espécie e a relação entre esses comportamentos e o ambiente onde o quero-quero se estabeleceu.

Agradecimentos. Agradecemos à Prefeitura Municipal de Torres por permitir a realização do trabalho dentro dos limites do município e ao LABSMAR/UFRGS pela infraestrutura que permitiu o desenvolvimento deste projeto. CJC recebeu bolsa do Programa Nacional de Pós-doutorado/ Coordenação de Aperfeiçoamento de Pessoal de Nível Superior.

\section{REFERÊNCIAS}

Altmann, J. 1974. Observational study of behavior: Sampling methods. Behaviour 49:227-267.

Ayres, M.; Ayres Junior, M.; Ayres, D. L. \& Santos, A. S. 2007. BioEstat 5.0: aplicações estatísticas nas áreas das ciências biológicas e médicas. Belém, MCT, IDSM, CNPq.

Bakhturina, D. S. \& Klenova, A. V. 2016. Quantitative analysis of the behavior of three auk species (Charadriiformes, Alcidae): Crested auklet (Aethia cristatella), parakeet auklet (Cyclorrhynchus psittacula), and horned puffin (Fratercula corniculata). Biology Bulletin 43(7):670-684.

Barnard, C. J. \& Stephens, H. 1981. Prey size selection by lapwings in lapwings/gull associations. Behaviour 77(1-2):1-22.

Beintema, A. J. \& Visser, G. H. 1989a. The effect of weather on time budgets and development of chicks of meadow birds. Ardea 77(2):181-192.

Beintema, A. J. \& Visser, G. H. 1989b. Growth parameters in chicks of charadriiform birds. Ardea 77(2):169-180.

Belton, W. 1994. Aves do Rio Grande do Sul, distribuição e biologia. São Leopoldo, Unisinos. 584p.

Buitron, D. 1983. Variability in the responses of black - billed magpies to natural predators. Behaviour 78:209-236 
Cestari, C. 2009. Tremular dos pés da batuíra-de-bando Charadrius semipalmatus: revelando presas na superfície de praias brasileiras. Biota Neotropical 9(4):299-301.

Coleman, D. C. \& Crossley JR, D. A. 1995. Fundamentals of soil ecology. San Diego, Elsevier. 376p.

Costa, L. C. M. 1994a. Comportamento agonístico de Vanellus chilensis (Wagler, 1827) (Aves, Charadriiformes) em Curitiba, Paraná, Brasil. Revista Acadêmica 9:21-30.

Costa, L. C. M. 1994b. Manobras de distração de Vanellus chilensis (Wagler, 1827) (Charadriiformes, Charadriidae) em Curitiba, Paraná, Brasil. Estudos de Biologia 3(36):33-42.

Costa, L. C. M. 2002. O comportamento interespecífico de defesa do quero-quero, Vanellus chilensis (Molina, 1782) (Charadriiformes, Charadriidae). Revista de Etologia 4:95-108.

Del Hoyo, J.; Elliott, A. \& Sargatal, J. 1996. Handbook of the Birds of the World. Barcelona, Lynx Edicions. 812p.

Del-Claro, K. 2004. Comportamento Animal - Uma introdução à ecologia comportamental. Jundiaí, Conceito. 132p.

Delfino, H. C. 2019a. [XC492891 · Southern Lapwing - Vanellus chilensis]. Xeno-canto. Disponível em <https://www.xeno-canto.org/492891>. Acessado em 16 ago 2019.

DeLFino, H. C. 2019b. [XC492892 · Southern Lapwing · Vanellus chilensis]. Xeno-canto. Disponível em $<$ https://www.xeno-canto.org/492892>. Acessado em 16 ago 2019.

Delfino, H. C. 2019c. [XC492895 · Southern Lapwing · Vanellus chilensis]. Xeno-canto. Disponível em <https://www.xeno-canto.org/492895>. Acessado em 16 ago 2019.

ELLIOT, R. D. 1985. The effects of predation risk and group size on the antipredator responses of nesting lapwings Vanellus vanellus. Behaviour 92(1-2):168-187.

Ellison, A. M. \& Gotelli, N. J. 2011. Princípios de estatística em ecologia. Porto Alegre, Artmed. 528p.

GaLBRAITH, H. 1989. Arrival and habitat use by lapwing Vanellus vanellus in the early breeding season. Ibis 131(3):377-388.

GRISSER, P. 1990. Anti-predator postures of lapwing and stonechat. British Birds 83(10): 425 .

Henrique, C. A. De M. \& Piratelli, A. 2008. Etograma de garça-brancagrande, Casmerodius albus (Ciconiiformes, Ardeidae). Revista Brasileira de Ornitologia 16(3):185-192.

HesP, L. S., \& Barnard, C. J. 1989. Gulls and plovers: age-related differences in kleptoparasitism among black headed gulls (Larus ridibundus). Behavioral Ecology and Sociobiology 24(5):297-304.

IVERSEN, F. M. 1986. The impact of disturbance on the lapwings Vanellus vanellus incubation. Dansk Ornitologisk Forenings Tidsskrift 80(34):97-102.

Kalsi, S. R., \& KHERA, S. 1987. Agonistic and distraction behaviour of the redwattled lapwing, Vanellus indicus indicus. Pavo 25(1-2):43-56.

KirbY, J. S., \& GreEN, R. E. 1991. Nest defence by black-tailed godwits on the Ouse Washes, Cambridgeshire, England. Wader Study Group Bulletin 61:71-72.

Kumar, A. \& Sharma, R. K. 2011. Observations on breeding behaviour and vocalizations in Red-wattled Lapwing, Vanellus indicus (Aves: Charadriidae) from Northern India. Journal of Experimental Zoology 14(1):333-338

LoREnz, K. 1995. Os fundamentos da etologia. São Paulo, Editora da Universidade Estadual Paulista. 466p.

Maclean, G. L. 1972. Problems of display postures in the Charadrii (Aves: Charadriiformes). Zoologica Africana 7:57-74.

Maruyama, P. K.; Cunha, A. F.; Tizo-Pedrozo, E. \& Del-Claro, K 2010. Relation of group size and daily activity patterns to southern lapwing (Vanellus chilensis) behavior. Journal of Ethology 28:339-344.

Marsh R. L. \& Dawson W. R. 1989. Avian Adjustments to Cold. In: Wang, L. C. H. eds. Animal Adaptation to Cold. Advances in Comparative and Environmental Physiology, vol 4. Berlin, Springer. 458p.
McMahon, N. 1996. Carrion crows killing several northern lapwings. Bristish Birds 89(6):278.

Moller, A. P. 2000. Male parental care, female reproductive success, and extrapair paternity. Behavioral Ecology 11(2):161-168.

Oliveira, H.; SouzA, D. R. A. \& Silva, M. 2014. Etograma do Carcará (Caracara plancus, Miller, 1777) (Aves, Falconidae), em cativeiro. Revista de Etologia 13:1-9.

Pillar, V. P; Müller, S. C; Castilhos, C. M. S. \& Jacques, A. V. A. eds. 2009. Campos Sulinos: conservação e uso sustentável da biodiversidade. Brasília, Ministério do Meio Ambiente. 403p.

Plummer, K. E; Bearhop, S; Leech, D. I; Chamberlain, D. E \& Blount, J. D. 2013. Winter food provisioning reduces future breeding performance in a wild bird. Scientific Reports 3:1-6.

Porto, G. R. \& Piratelli, A. 2005. Etograma da maria-preta, Molothrus bonariensis (Gmelin) (Aves, Emberizidae, Icterinae). Revista Brasileira Zoologia 22(2):306-312.

R CORE TEAM. 2015. R: A language and environment for statistical computing. R Foundation for Statistical Computing, Vienna, Austria. Disponível em $<$ https://www.R-project.org/>.

Ridgely, R. S. Y \& GREENField, P. J. 2001. The birds of Ecuador. Volume 1. Status, distribution and taxonomy. Ithaca, Cornell University Press. 880 p.

SANTos, E. S. A. 2010. Southern Lapwing (Vanellus chilensis), version 1.0. In: Schulenberg, T. S. ed. Neotropical Birds Online. Ithaca, Cornell Lab of Ornithology. Disponível em $<$ https://doi.org/10.2173/ nb.soulap1.01>. Acesso em 10 nov 2017.

SAntos, E. S. A. \& Macedo, R. H. 2011. Load lightening in Southern Lapwings: group living mothers lay smaller eggs than pair-living mothers. Ethology 117:547-555.

SARACURA, V.; Macedo, R. H. \& BlomQvist, D. 2008. Genetic parentage and variable social structure in breeding Southern Lapwings. Condor 110:554-558

SICK, H. 1997. Ornitologia Brasileira. Rio de Janeiro, Nova Fronteira. 862p.

SimON, J. E. \& PACHECO, S. 2005. On the standardization of nest descriptions of neotropical birds. Revista Brasileira de Ornitologia 13(2):143-154

Thomas, D. H. 1983. Aposematic behaviour in the blacksmith plover. Ostrich 54(1):51-52.

Thompson, D. B. A. 1983. Prey assessment by Plovers (Charadriidae): net rate of energy intake and vulnerability to kleptoparasites. Animal Behaviour 31:1226-1236.

Thompson, D. B. A. 1986. The economics of kleptoparasitism: optimal foraging, host and prey selection by gulls (Larus ridibundus). Animal Behaviour 34(4): 1189-1205.

Thompson, D. B. A. \& Barnard, C. J. 1983. Antipredator responses in mixed-species associations of lapwings (Vanellus vanellus), golden plovers (Pluvialis apricaria) and black-head gulls (Larus ridibundus). Animal Behaviour 31(2):585-593.

Thompson, D. B. A. \& Barnard, C. J. 1984. Prey selection by plovers (Pluvialis apricaria): optimal foraging in mixed-species groups. Animal Behaviour 32(2):554-563.

Thompson, D. B. A. \& Lendren, D. W. 1985. Gulls and plovers: host vigilance, kleptoparasite success and a model of kleptoparasite detection. Animal Behaviour 33(4):1318-1324.

WALTERS, J. R. 1979. Interspecific aggressive behavior by long-toed lapwings (Vanellus crassirostris). Animal Behaviour 27(4):969-981.

Walters, J. R. 1982. Parental behavior in lapwings (Charadriidae) and its relationships with clutch sizes and mating systems. Evolution 36:1030-1040

WARD, D. 1989. Behaviour associated with breeding of crowned, blackwinged and lesser blackwinged plovers. Ostrich 60(4):141-150.

WiLSON, E. O. 1975. Sociobiology: the new synthesis. Cambridge, Massachusetts. Belknap Press of Harvard University Press. 697p. 